\title{
Marcos de referencia en el estudio de los mapas cognitivos de ambientes urbanos
}

\section{Ignacio Aragonés}

Departamento de Psicología Social

(Fac. Psicologia) Universidad Complutense (Madrid)

La preocupación por los temas ambientales junto con la orientación más «vigente» en la Psicología, lo cognitivo, ha supuesto que la cognición ambiental haya tomado gran importancia en la literatura psicológica de los últimos tiempos. Dentro de este área, el estudio de los mapas cognitivos de ambientes urbanos, sin lugar a duda, ha sido el constructo más investigado. A partir de la obra de Lynch (1960) "La imagen de la ciudad» y en estas dos décadas largas la producción sobre este tema ha ido en aumento fundamentalmente hasta finales de los años setenta, pero, a pesar de la múltiple realización de trabajos publicados, no existe un marco teórico lo suficientemente sólido que sea capaz de articular todos los conocimientos obtenidos (Downs y Stea, 1973; Holalan y Dobrowdlny, 1978; Evans', 1980).

Este articulo pretende recoger las aportaciones más relevantes que se han venido sucediendo en este campo. Para ello se ha recurrido a trabajos de autores importantes en la materia, presentados por orden cronológico de publicación. Dentro de cada uno se exponen los hallazgos fundamentales de cada uno de ellos y se señalan, igualmente, aquellos estudios que enriquecen o critican las aportaciones "originales».

\section{Lynch (1960)}

Es el primer trabajo realizado sobre el tema y fuente de inspiración de todos los trabajos posteriores. Hasta ese momento no se habia realizado ningún trabajo sobre la imagen de la ciudad que no echara mano de la Historia, y es con Lynch, un planificador urbano, con el que el tema de los mapas cognitivos en los ambientes de grandes dimensiones empieza a estudiarse, aunque el autor llamó al constructo «imagen de la ciudad». Comenzar por esta obra es obligatorio, no sólo por ser 


\section{Estudios}

la primera, sino también por ser una de las aportaciones más fructíferas que existen en este campo, tal y como indica Crane (1961), un año después de la aparición de "La imagen de la ciudad" (tal apreciación sigue siendo confirmada en los escritos más recientes). La forma urbana ya se puede estudiar desde una perspectiva psicológica, es decir de forma "subjetiva» y no sólo de forma "objetiva", como se hace en la cartografia (Boaga, 1977). No hay acuerdo entre los distintos autores acerca de la orientación que utilizó Lynch para estudiar las imágenes de las ciudades. Así, Sica (1977) sitúa este primer trabajo entre la escuela de la Gestalt y el estructuralismo lingüistico y, por poner otro ejemplo, Stringer (1978) dice que, aunque evoca de alguna forma la Gestalt, en realidad está entroncado con los aspectos más pragmáticos de la planificación. En cualquier caso, reconoce la gran importancia del trabajo como aportación de unas técnicas para analizar la percepción ambiental.

El estudio empirico que Lynch realiza tiene como objetivos fundamentales, según sus propias palabras, desarrollar ideas y métodos acerca de cómo se forman las imágenes urbanas, cómo se pueden medir y cuáles son sus contenidos. La investigación ha sido realizada con un escaso número de personas - sesenta - en las ciudades de Boston, Jersey City y Los Angeles.

Comienza su obra con el intento de descifrar cómo se percibe una ciudad y respondiéndose a esto manifiesta: «a menudo, nuestra percepción de la ciudad no es continua sino, más bien, parcial, fragmentaria, mezclada con otras preocupaciones. Casi todos los sentidos están en acción y la imagen es la continuación de todos ellos». Como se podrá ver a simple vista, en este párrafo se encuentran aspectoś que en años posteriores destacarán otros estudiosos sobre el tema, tales como la "sobrecarga del sistema» de Mil- gram (1970) y el «filtrado de la información ambientaly de Bailly (1979); así como el reconocimiento explícito de la intervención de diversos sentidos en la formación de la imagen urbana. Aun sin hacer ninguna referencia a Tolman (el defensor del aprendizaje de los mapas cognitivos), plantea la improbabilidad de que exista un instinto para formar la imagen y opina que más bien será la organización y el uso de ciertas claves lo que permite formalizar a cada persona su propia imagen, destacando la influencia de los aspectos transculturales.

Partiendo del supuesto de que existen imágenes urbanas individuales, es decir la imagen de la ciudad que tiene cada persona, plantea la probabilidad de «imágenes públicas» es decir: «representaciones comunes que hay en grandes números de habitantes de una ciudad. Se trata de los puntos de coincidencia que puede esperarse que aparezcan en la interacción de una realidad fisica única, una cultura común y una naturaleza fisiológica básica». En esta misma línea se encuentran geógrafos como Pockoc (1972) y psicólogos como Milgram (1977). Este tipo de imágenes es la que dice ser de gran utilidad para los urbanistas, si bien autores como Bell, Randal y Roeder (1973) señalan que el trabajo de Lynch no ha sido muy utilizado por los diseñadores. En la misma línea Appleyard (1969) observa la dificultad de valorar estos hallazgos por los planificadores.

Lynch atribuye a las imágenes ambientales tres componentes: identidad, estructura y significado, imbricados en los estímulos. Conviene señalar, en este momento, que Lynch estudia de forma empírica las dos primeras propiedades, por estimar que el significado de los elementos es heterogéneo en la población de una gran ciudad y, por tanto, de difícil inclusión en la imágen pública de la misma.

Para que un sujeto pueda componer una imagen ambiental que le permita 
tener buen sentido de la orientación dentro de la ciudad recesita de la obtención de muchas claves que configuren su imagen. Lynch, a partir de la identidad y la estructura, define la «imaginabilidad» o «legibilidad» de un elemento urbano como la "probabilidad" de suscitar una imagen en la persona, o (como dice en otro lugar hablando de la «legibilidad del paisaje urbano") es la "facilidad con que se pueden reconocer y organizarse sus partes en una pauta coherente».

A través de las observaciones, entrevistas y dibujos de mapas analiza los objetos fisicos de la ciudad y sugiere que la imagen de la ciudad está compuesta por elementos organizados en cinco categorias: sendas, bordes, barrios, nodos y mojones. Esta clasificación ha sido contrastada empíricamente por Magaña (1978) y Aragonés y Arredondo (en prensa), lo que avala la validez de este modelo, que ha venido siendo admitido de forma sistemática por todos los autores, si bien Norberg-Shulz (1971), Goodey et al., (1971) y Beck y Wood (1976) han sugerido una clasificación más simple y que plantease menos problemas. Se trata de reducir las cinco categorías de Lynch a tres: punto o foco, línea y área, como elementos que componen el mapa cognitivo, aunque sin excesivo eco.

Un trabajo ligeramente posterior al de Lynch, y que pretende comprobar la eficacia de sus técnicas, es el llevado a cabo por De Jonge (1962), otro arquitecto. Investiga en las ciudades europeas de Amsterdam, La Haya y Rotterdam, confirmando la utilidad del dibujo del mapa de la ciudad realizado por los sujetos, encontrando las cinco categorías y estableciendo como conclusión que las ciudades con una imagen clara son aquéllas que tienen una definida estructura espacial, necesitando menos mojones que cuando la estructura es vaga; en este último caso se precisan múltiples detalles para conformar una imagen eficaz en la orientación o toma de decisiones espaciales.

Para terminar con esta breve presentación del trabajo de Lynch quizá convenga comentar aquel aspecto que le ha sido criticado: el no haber estudiado los aspectos referidos al significado de los estímulos ambientales, tan relevante en el conocimiento de la ciudad como ha puesto de manifiesto Wood (1971), según comentarios hechos por Saarinen (1976) y Stringer (1978). Dice este autor que no sólo son importantes los aspectos físicos («urbs»), sino también los de orden social, político y recreativo ("civitas»), produciéndose una reproducción en los niveles casa, barrio y ciudad. Otro aspecto que si bien no tiene que ver con el mapa cognitivo propiamente dicho sí está en intima relación con él, es el «esquema» de Francescato y Mebane (1973). Este concepto recoge aspectos tales como «la organización conceptal de la ciudad, símbolos, creencias y actividades». Por tanto, se pude decir, como señala Appleyard (1979), que un elemento urbano forma parte de la imagen de un sujeto, en función del significado social y funcional, y conjuntamente con la imaginabilidad que éste posea; tal y como fue verificado empíricamente por Gulick (1963) y Appleyard (1976).

A modo de resumen se puede concluir que la aportación de Lynch al estudio de los mapas cognitivos de los ambientes urbanos es de primera magnitud por ofrecer un método de investigación y una clasificación de los contenidos de aquéllos. Como crítica se le puede achacar la no incorporación del significado del estímulo ambiental al método e investigación.

\section{Klein (1967)}

Señalar bajo un epígrafe la investigación de este autor es obligatorio a la vista de la importancia que la percepción del 
centro de la ciudad tiene para la conformación de la imagen de toda ella, tal y como señalan numerosos autores (De Jonge, 1962; Siervets, 1967; Goodey et al., I971; Ross, 1974; Bailly, 1979; Lee, 1976; Shimizu, 1979; Aragonés, 1983). El modelo que Klein presenta en su investigación sobre la ciudad de Karlsruhe es confirmado por Zawawi (1970) y Saarinen (1976) en las ciudades de Washington y Chicago, respectivamente; igualmente, es valorado por Rapaport como «el estudio más completo de la definición subjetiva de un centro de ciudad".

El objetivo de su investigación es estudiar de forma empírica qué imagen del centro de la ciudad tiene una muestra de sus habitantes —algo más de I.000 sujetos- a través de la opinión que emitian de si pertenecían al centro o no un total de 24 elementos - sendas y nodos-, cuyos nombres se imprimían en otras tantas tarjetas. Diferencia dos tipos de estructuras dentro de la ciudad: «la topográfica», que se refiere al aspecto geográfico del centro de la ciudad, y la ufuncional", al tipo de actividad que se realiza en cada uno de los elementos que se presentan como estímulos.

A partir de los resultados empíricos obtiene una serie de conclusiones que, si bien no se pueden generalizar como imagen del centro de la ciudad, sí tienen al menos un carácter indicativo para las próximas investigaciones empíricas.

Las conclusiones más relevantes son las siguientes:

- La localización de la vivienda influye: cuanto más en el centro viven los sujetos más pequeño es éste. $\mathrm{El}$ centro se estira hacia la zona de la vivienda, lo que supone aproximar el centro al hogar.

- El sexo influye de forma tal que las mujeres poseen el centro más grande que los hombres; las primeras lo fijan en función de tiendas elegantes y centros culturales y los hom- bres en función de los edificios de la Administración.

- El tiempo de residencia influye de forma directamente proporcional, hasta un máximo.

- La profesión influye de forma inversa: cuanto más alto es el status profesional más estrecho es el centro de la ciudad, igualmente ocurre con el nivel de educación.

- No se observan diferencias con otras variables tales como la edad adulta, la motorización o el nacimiento en la ciudad.

En otras investigaciones sobre la imagen del centro, tales como la de Sieverts (1967), se comprueba que el centro de la ciudad se percibe sobredimensionado. En esta misma linea, Ross (1974) afirma que generalmente el centro es imaginado más grande y las afueras más pequeñas que la realidad.

\section{Appleyard (1969) (1970) (1976)}

En el año 1976 aparece una monografía sobre la investigación que realizó este planificador en la ciudad de Guayana (Venezuela), donde se completan las publicaciones de los años 1969 y $197^{\circ}$ con más detalle y mayor número de datos. Como en los casos anteriores, los objetivos son fundamentalmente empiricos, pero a partir de los datos establece una serie de conclusiones, entre las que destacan las categorias acerca del mapa cognitivo de la ciudad, denominados «estilos cognitivos".

La investigación la lleva a cabo con 2 I I sujetos de diversos distritos de la ciudad y un grupo de ingenieros y planificadores. La técnica utilizada fue el dibujo del mapa de la ciudad y de una zona de ésta. Este método de recogida de información es criticado por el mismo autor, debido a la dificultad que la ejecución de la prueba supone para algunos 
sujetos; hecho que ha sido puesto de manifiesto también por otros autores (Francescato y Mebane, 1973, y Magaña, 1978). Sin embargo estima importante el dibujo del mapa por tener la capacidad de mostrar cómo los sujetos estructuran la ciudad, si bien los aspectos como el simbolismo, imagen visual y significado no aparecen prácticamente reflejados. $Y$ así manifiesta que a través de esta técnica no se obtiene un entendimiento completo de la ciudad.

En cuanto a los resultados más relevantes caben destacarse la confirmación de algunos de los obtenidos por Lynch ( 1960 ) como la observación de diversos grupos de exactitud, desde aquellos que eran desconexionados hasta los que eran fuertemente conexionados. Aspecto este que será tratado posteriormente a la luz de la investigación de Moore (1973). El establecimiento de los denominados «estilos cognitivos" lo realiza a través de un análisis de contenido de los mapas, fijando dos grandes grupos:

Secuenciales: mapas formados fundamentalmente por calles y nodos.

Espaciales: mapas formados fundamentalmente por barrios, mojones y límites.

A su vez, cada uno de éstos se subdivide en cuatro tipos, de menos a más complejo y exacto. Como resultado global se puede señalar que el 75 por 100 optó por la estructura secuencial, lo que explica en cierta manera la importancia de la circulación según indica Appleyard. Señala, igualmente, algunas variables influyentes en la formación del mapa, tales como familiaridad con la ciudad, tiempo de residencia, formas de viajar y, sobre todo, las zonas de uso como el lugar de la vivienda, el trabajo, compras y algunos lugares ocasionales.

Por último se observan grandes diferencias entre la percepción de la ciudad que hace un planificador o técnico en urbanismo y los usuarios, preocupación sobre la que vuelve Appleyard (1977).
Otra clasificación similar es la realizada por Sieverts (1967) en una investigación que realiza con estudiantes en la ciudad de Berlín; distingue dos tipos de estructuras en los dibujos: "mapa-imagen» (es decir, una estructura más o menos compleja de la ciudad) e «imágenes puramente derivadas de la experiencia de pasear o conducirm. Pero reconoce una combinación de ambos tipos en la mayoría de los mapas recogidos.

\section{Goodey (1971)}

Los geógrafos, obviamente, también se han preocupado de explicar la formación del mapa cognitivo referido a los ambientes urbanos, pero no se han fijado tanto en los procesos mentales como en los aspectos espaciales, o mejor dicho en el tipo de comportamientos que realizan los sujetos en determinados lugares. El hecho de abrir un epígrafe con Goodey, no responde más que a razones cronológicas, pues geógrafos destacados, como Norberg-Schulz (1971), Pockock y Hudson (1978) y Bailly (1979) siguen modelos funcionales, idénticos o similares.

Goodey (1971) establece dos tipos de fuentes diferentes que influyen en el desarrollo de las imágenes ambientales:

Primarias: derivadas de la experiencia del ambiente (compras, ocio, trabajo, amigos y experiencias personales).

Secundarias: mass-media y contactos personales.

Por otra parte, Bailly (1979), en su tesis de estado presentada en 1977 en París, conforma un modelo de percepción de la ciudad a partir de la experiencia del medio, centrándose en torno al siguiente esquema: áreas alrededor de la vivienda, trabajo y residencia de amigos, percepción del centro subjetivo de la ciudad, lugar de compras y segunda referencia, así como la acumulación de informaciones que la persona realiza en los pasillos a través 


\section{Estudios}

de los cuales se desplaza para ir de un lugar a otro.

En diversas investigaciones se resaltan algunos de estos aspectos que ya han sido mencionados, pero quizá convenga citar aqui los tres experimentos de Evans y Pezdek (1980), donde se confirma la importancia que tiene la experiencia directa en el conocimiento de los ambientes. Igualmente, Karan, Bladen y Singh (1980), en la investigación de un barrio pobre de la ciudad de Ptna (India), observan la influencia de los lugares de trabajo y residencia en el mapa mental, al igual que el área central de la ciudad frente al área metropolitana.

Estas explicaciones dadas por los geógrafos están concebidas desde la perspectiva de que la conducta determina el mapa cognitivo, pero olvidan lo opuesto; es decir, que el proceso de crecimiento de un mapa afecta también al comportamiento, existiendo permanentemente una interacción mutua conducta-mapa cognitivo (Orleans, 1973).

\section{Milgram et al. (1972)}

Las investigaciones de Milgram et al. (1972) y Milgram y Jodelet (1977), en las ciudades de Nueva York y París, respectivamente, son dos hitos en el estudio de los mapas cognitivos de los ambientes urbanos, en cuanto a la utilización de diapositivas como técnica de investigación y a la formulación sobre el reconocimiento de un elemento urbano, en la primera de ellas.

Parte de una concepción durkheniana, es decir, la ciudad es un hecho social; si bien dice que no debe ser sólo tratada como tal, sino también la percepción de la misma. Afirma que uuna ciudad es más una representación colectiva que un conjunto de calles, plazas y edificios» (Milgram y Jodelet, 1977). En esta misma línea un arquitecto español, Chueca
(1974), comentando algunos aspectos históricos y urbanísticos de la ciudad de Madrid, dice que las «ciudades son entes vivos rebosantes de personalidad».

La forma que una persona tiene de conocer dónde se encuentra, en un contexto ambiental, la presenta como el paradigma fundamental del «mapa psicológico", o lo que es lo mismo, mapa cognitivo, precisando de tres requisitos la localización del lugar:

- Ser diferente de otros de la ciudad.

- Ser único respecto a los situados en la memoria del sujeto.

- Situarlo en un marco de referencia.

A pesar de reconocer que los mapas cognitivos no son sólo el reconocimiento de escenas urbanas, sino que éstas son una parte del proceso, su primera investigación en la ciudad de Nueva York es realizada con este enfoque; pidió a 200 sujetos que reconociesen 1 I 2 diapositivas, de otros tantos puntos, tomados de forma aleatoria a través de las intersecciones que se producen en una cuadrícula de r.000 metros de paso, en cinco barrios de la ciudad. Con este método, más estructurado que el de Lynch (1960), llega a conclusiones similares a las ya señaladas anteriormente: el lugar de residencia y el centro de la ciudad influyen en la formación del mapa cognitivo.

Por otra parte, llega a establecer una relación multiplicativa entre dos variables -centralidad con respecto al flujo de población (C) y características sociales o arquitectónicas (D)-, que le permiten fijar el grado en el cual una escena de la ciudad puede ser reconocida.

$$
R(\text { reconocimiento })=f(C \times D)
$$

En ningún momento fija el tipo de función que permite que una escena sea más o menos reconocida. Pearce y Carney (1980), con técnica similar (reconocimiento de diapositivas de la ciudad de Adelaida), establecen que hay una relación entre 
centralidad del flujo de población y familiaridad con el lugar, de tal forma que ambas variables están indisolublemente unidas. Este último aspecto, la familiaridad, es resaltado por otros autores como Golledge, Briggs y Denko (1969), Ladd (1970), Pocok (1972), Francescato y Mebane (1973).

Investigaciones empíricas realizadas en otras ciudades resaltan la gran importancia que el uso del elemento arquitectónico tiene en su reconocimiento, tal y como señalan Sieverts (1967) en Berlín y Francescato y Mebane (1973) en Roma y Milán. No obstante, los monumentos turísticos son muy recordados por los sujetos aunque no sean usados, como se demuestran en las investigaciones anteriores y en la realizada por Milgram y Jodelet (1977) en París. Parece que estos elementos son reconocidos en función del significado que tienen en el marco social de la ciudad. En esta línea, Milgram et al. (1972) señalan que un grado de alta precisión de un mapa cognitivo de un medio urbano no implica sólo dar, reconocer y estructurar los diversos símbolos; sino también el grado preciso de significado que cada uno de los puntos tiene con respecto a los demás.

Por último, conviene señalar que en el reconocimiento de una escena urbana pueden influir los medios de comunicación de masas, como ponen de manifiesto Moore y Golledge (1976). En esta línea existe un trabajo sobre la ciudad de San Francisco realizado por Kreimer (1.973), en el que se estudia la imagen de la ciudad que crea un periódico a través de un análisis de contenido.

\section{Kaplan (1973 a) (1973 b)}

Este autor elabora un marco integrado, establece la necesidad de conocer el ambiente y la de tener un mapa cognitivo para poder supervivir en las cooordena- das espacio-temporales. También distingue cuatro categorías o dominios de procesamiento que corresponden al contacto con el ambiente:

Reconocimiento: conocer dónde se encuentra uno y reconocer los objetos del ambiente.

Predicción: conocer lo que puede suceder.

Evaluación: conocer si las cosas son buenas o malas, siendo posible anticipar si las acciones alternativas tienen consecución probable.

Acción: conocer el qué hacer, pudiendo pensar en posibles alternativas.

Estos cuatro dominios son considerados por Kaplan como necesidades humanas. Por tanto el mapa cognitivo, Lay-out de la ciudad, o su aspecto geométrico, no es más que una parte de lo que el autor establece como campos de conocimiento, su «mapa cognitivo", tal y como comenta Cadwalleder (1979). Por otra parte, en este tipo de estudios existe una cierta tendencia a estudiar tan sólo el primer aspecto, sin llegar a investigar empíricamente la influencia en la toma de decisiones espaciales (Evans, I980).

\section{Moore (1973) (1974)2 (1975)}

El trabajo realizado por este autor a lo largo de la década de los años 70 , solo o en colaboración con otros autores, supone la formalización del estudio de los mapas cognitivos desde una perspectiva evolutiva (1973) (1974) (1975) (1976). Distingue tres niveles distintos de desarrollo:

Nivel I: Egocéntrico e Indiferenciado.

Nivel II: diferenciado y parcialmente coordinado.

Nivel III: abstractamente coordinado y jerárquicamente integrado.

Los tres niveles fueron fijados en función del grado de familiaridad que decían tener los sujetos de la ciudad o de un 
barrio, observándose que cuanto más familiaridad tenían los sujetos, existía un mayor nivel de desarrollo en la formalización del mapa cognitivo.

Por otra parte, no se encontraron diferencias significativas con la edad, sexo e inteligencia en general, pero sí con el nivel de instrucción, las habilidades espaciales y la conducción de vehículos.

En linea similar se puede situar a Metton (1974), quien distingue entre mapas «egocéntricos» y "complejos y no egocéntricos", observando la influencia de aspectos sociales y de personalidad. Igualmente Hardwick et al. (1976) sugieren que los adultos tienen multiniveles de representación del ambiente espacial: representación de relaciones ordinales entre las formas y subrepresentaciones más pequeñas, con un punto de vista particular.

\section{Holaban y Dobrowdlny (1978)}

Estos autores insisten sobre la carencia de modelos teóricos que expliquen la formación de los mapas cognitivos, destacando, fundamentalmente y por el momento, los trabajos descriptivos. En un intento de explicar la formación del mapa cognitivo, realizan una investigación tomando como marco ambiental un campus universitario, para la que utilizan una combinación de métodos, como indica Stockls (1978): dibujos de mapas, un pequeño cuestionario y observación sistemática de cierto lugares del campus.

Establecieron como conclusión de su investigación que los universitarios forman sus mapas cognitivos en función de dos variables: «las conductas individuales» y «las conductas colectivas» que realizan en el campus, confirmándose las siguientes hipótesis: a) «Los límites y formas espaciales incluidos en los mapas cognitivos están directamente relacionados con la conducta individual y colectiva."

b) «La exageración del tamaño de las formas espaciales en los mapas cognitivos y la dislocación del centro del campus está directamente relacionada con las diferencias individuales en la conducta personal en el ambiente.»

Sugieren la necesidad de investigar el problema a nivel de la ciudad, teniendo en cuenta el estilo de vida urbana según los estudios de Michelson (1975).

\section{COMENTARIO FINAL}

Los estudios comentados ponen de manifiesto las dificultades de articular los conocimientos sobre mapas cognitivos dentro de único modelo, posiblemente debido a que todos ellos han sido producto de estudios empiricos sin apenas sustrato especulativo. No obstante, se puede finalizar que tras las revisiones críticas hechas sobre el tema por Moore (1979) y Evans (1980) ${ }^{1}$ se está en condiciones de poder comenzar en una línea de trabajo donde lo que prime sean los aspectos integradores frente al puro dato empírico.

En este dossier se ha incluido un artículo de Moore en que se expone una investigación concreta sobre la formación y el desarrollo de los mapas cognitivos en adolescentes. Para completar la perspectiva evolutiva se han incluido también otros dos artículos: uno de Alvaro Marchesi sobre conceptos espaciales, mapas cognitivos y orientación en el espacio, y el segundo, de Esperanza Ochaíta, sobre la teoría piagetiana acerca de la génesis de la representación espacial.

\section{Notas}

1 Este articulo responde a una de las mejores revisiones hechas sobre el tema de la cognición ambiental, lo que ha merecido su inclusión en la composición del dossier. 


\section{Referencias}

APPLEYARD, D. D.: "Why buildings are knowing a predictive tool for architects and planners». Environment and Bebavior, 1969, 1, 131-156.

APPLEYARD, D. D.: «Styles and Methods of Structuring a City». Environment and Bebavior, $1970,2,100-117$. Appleyard, D. D.: Planning a Pluralist City: Conflicting Realities in Ciudad Guayana, MIT Press, Cambridge, Mass., 1976.

APPLEYARD, D. D.: “A Planner's guide to environmental Psychology: A review essay». J. American Institute Planners, 1977, 43, 184-189.

APPIEYARD, D. D.: "The environnmental as a social symbol». Journal of the American Planning Association, $1979,45,143-153$.

Aragones, J. I.: Mapas cognitivos de Ambientes Urbanos: Un estudio empirico sobre Madrid, Tesis Doctoral, Universidad Complutense, Madrid, $19^{83}$.

ARAgones, J. I., y ARREDONDO, J. M.: "Structure of Urban cognitive Maps». Journal of Environmental Psychology, (en prensa).

BAll.ly, A. S.: Le percepción del espacio urbano. Conceptos, métodos de estudio y su utlización en la investigación urbanistica, Instituto de Estudio de Administración Local (Nuevo Urbanismo, 29), Madrid, 1979.

BECK, R. J., y WOOD, D.: “Cognitive Transformation of information from urban geographie fields to mental maps). Environment and Behavior, 1976, 8 (2), 199-238.

Bel.I., G.; RANDAll, E., y ROEDER, J.: Urban environments and buman behavior: An annotated bibliography, Dowden, Hutchinson y Ross, Stroundsburg, P. a, 1973.

BOAGA, G.: Diseño de tráfico y forma urbana, Gustavo Gili, Barcelona, 1977.

CADWAllader, M.: "Problems in cognitive distance implication for cognitive mapping". Environment and Behavior, 1979, I1 , 4, 576-579.

Chueca, F.: Madrid, ciudad con vocación de capital, Ed. Pico Sacro, Santiago de Compostela, 1974.

CRANe, D. A.: «Review of the Image of City". Journal of the American Institute Planner, 1961, $27,152-194$.

DE JONGE, D.: «Images of urban areas: their structure and psychological foundations». Journal of American Institute Planners, 1962, 28, 266-276.

Downs, R. M., y STEA, D.: «Cognitive maps and spatial behavior: Process and products». En R. M. Downs y D. Stea: Image and Environment, Aldine, Chicago, 1973.

Evans, G. W.: «Environmental Cognition». Psychological Bulletin, 1980, 88 (2), 259 - 287.

EVANS, G. W., y PEZDEK, K.: “Cognitive mapping: Knowledge of real-world distance and location information". Journal of Experimental Psycbology: Human leargning and memory, 1980, 6, 13-24.

Francescato, D., y Mebane, W.: "How citizens view two great cities: Milan and Rome». En R. M. Downs, y D. Stea (eds.): Image and environment: Cognitive mapping and Spatial Bebavior, Aldine, Chicago, 1973.

GoLleDGE, R. G.: «Multidimensional analysis in the study of environmental behavior an environmental design". En Altman y J. Wohlwill (eds.): Human bebavior and environment (vol. 2), Plenum Press, Nueva York, 1977 .

GOODEY, B.: Perception of the Environment: An Introduction to the Literature, Ocasional Paper, núm. 17, Center for Urban an Rgional Studies, University of Birmingham, Birmingham, 1971.

GOOEDY, B. et al.: City-Scene. An Exploration into the Image of Central Birmingbam as Seen by Area Residents, Center for Urban and Regional Studies, University of Birmingham, 1971.

Gul.ICK, J.: «Images of an Arab city». Journal of the American Institute of Planners, 1963, 179-198.

HardwiCK, D. A.; Mcintyre, C. W., y PICK, H. L.: «The content and manipulation of cognitive maps in children and adultsm. Monograph of the society for Research in child development, 1976, 41 (3, serial núm. 166).

Hol.ahan, CH. J., and Dobrowlny, M. B.: "Cognitive and Behavioral correlates of the spatial environment, Environment and Bebavior, 1978, 10, 3, 317-333.

Kaplan, S.: “Cognitive Maps, Human Needs and the Designed Environment». En W. F. E. Preiser (ed.): Environmental Design. Researcb, I, Hutchinson and Ross, Stroudsburg, 1973 a.

KAPI,AN, S.: "Cognitive maps in perception and thought». En R. Downs y D. Stea (Eds.): «Image and environment». Cognitive mapping and spatial behavior, Aldine, Chicago, $1973 \mathrm{~b}$.

KARAN, P. P.; BI.ADEN, W. A., y SINGH, G.: "Slum Dwellers' and squatlers' images of the city". Environment and Bebavior, 1980, 12 (I), $81-100$.

KLEIN, H. J.: «The delimitation of the town center. The Image of Its Citizens. En Univesity of Amsterdam. Sociografical Departament». Urban core an Inner City, Leyden, E. J. Brill, 1967.

KREIMER, A.: «Building the imagery of San Francisco. An analysis of controversy over high-rise development, 1970-71\%. En W. F. E. Preisser (ed.): Environment Desing Research, II, Dowden, Hutchinson and Ross, Stroudsburg, 1973.

LADD, F. C.: «Black yonths View their Environment: Neighborhood maps». Environment and Bebavior, 1970, 2, 74-79.

LEE, T. R.: Psychology and the Environment, Gral. Editor Peter-Herriot, Londres, 1976.

LYNCH, K.: The Image of the City (versión castellana: La imagen de la ciudad, $4 .^{2}$ ed., Ediciones Infinito, Buenos Aires, 1976), MIT, Press, Cambridge, I960.

MagAÑA, J. Z.: "An empirical and Interdisciplinary Test of a Theory of Urban Perception» (Doctoral Dissertation, University of California, Irvine). Dissertation Abstracts International, 39, $1460 \mathrm{~b}$ (University Microfils, núm. $7^{8-1}$,, 840 ), 1978. 
Metron, A.: «L'espace perçu: diversité des approches». L'espace Geographique, 1974, 3, $228-229$.

Milgram, S.: "The experience of living in cities». Science, 1970, $167,1461-1468$.

Milgram, S. et al.: "A psychological map of New York city". American Scientist, 1972, 6o (2), $194^{-200 .}$

Milgram, S., y JoDelet, D.: «Psychological maps of Paris». En S. Milgram (ed.): The individual in a Social World.Essays and Experiments, Addison-Wesley Pub, Londres, 1977.

MoORE, G. T.: «Developmental differences in environmental cognition». En W. Preiser (ed.): Environmental design Researcb, Dowden, Hutchinson \& Ross, Stroudsburg, Pa., 1973.

MOORE, G. T.: «The development of environmental knowing: An overview of an interactional -constructi- vist theory and some data within-individual development variations". En D. Canter y T. Lee (eds.): Psycbology and the built environment, Architectural Press Ltd, Tonbrigde, 1974.

MOORE, G. T.: «Spatial relations ability and develolpmental levels of urban cognitive mapping: a research note». Man-Environment Systems, 1975, 5, 247-248.

MOORE, G. T.: "Theory and research on the development of environmental knowing". En G. T. Moore y R. G. Golledge (eds.): Environmental knowing. Theories Research, and Methods, Dowden, Hutchinson and Ross, Stroudsburg, Pa., 1976.

MOORE, G. T.: «Knowing about environmental knowing: The current state of theory and Research on Environmental cognitions. Environment and Bebavior, 1979, 1 I (1), 33-70.

MoORE, G. T., y GolledGe, R. G.: «Environmental knowing: Concepts and theories». En G. T. Moore y R. G. Golledge (eds.): Environmental knowing: Tbeories, Researcb and metbod, Dowden, Hutchinson \& Ross, Stroudsburg, 1976.

NoRberg-SHulz, C.: Existence, Space and Architecture, Studio Vista, Londres, 1971 .

Pearce, P., y Cairney, P.: «Recognizing city scenes: a test of Milgram's formula». International Journal of psycbology, 1980, $15,95-103$.

POCOK, D.: "The city of the mind: a review of mental maps in urban areas». Scottisb Geogrpbical Journal, $1972,88,116-124$.

POCOCK, D., y HUDSON, R.: Images of the Urban Environment, Columbia, U. Press, Nueva York, 1978.

RAPOPORT, A.: Aspectos bumanos de la forma bumana. Hacia una confrontación de las ciencias sociales con el diseño de la forma urbana, Gustavo Gili, Barcelona, $197^{8}$.

ROSS, H.: Bebavior and perception in strange environments, George Allen \& Unwin, Ltd., London, 1974.

SaARinen, T. F.: Environment and Plannig, Perception and Behavior, Houghton Miffin Company, Boston, M. A., 1976.

SiCA, P.: La imagen de la ciudad. De Esparta a Las Vegas, Gustavo Gili, Barcelona, 1977.

SHIMizU, H.: The structure of city image, Gihodo, Tokio, 1979.

SiEverTS, T.: «Perceptual Images of the city of Berlin». Urban Core and Imer city, Leiden Brill, University of Amsterdam, Sociografical Departament, 1967.

Stokols, D.: «Environmental Psychology». Annual Rev. Psycbology, 1978, 29, 253-295.

STRINGer, P.: «La comprensión de la ciudad». En D. Canter y P. Stringer (eds.): Interacción Ambiental, aproximaciones psicológicas a nuestros entornos físicos, Instituto de estudios de la Administración Local, Madrid, 1978.

Tot.man, E. C.: «Cognitive maps in rats and men». Psychological Review, 1948, 55, 189 -208.

WOOD, D.: Fleeting glimpses: Adolescent and other images of the entity called San Cristóbal de las Casas, Tesis doctoral inédita, Clark University, Chiapas, México, 1971.

Zawami, M.: Perception of Downtown: $A$ case study of Washington, Dc., Master's thesis George Washington University, 1970 .

\section{Resumen}

El presente articulo ofrece una revisión de los estudios realizados en las dos últimas décadas sobre mapas cognitivos aplicados al mundo urbano a partir de las investigaciones llevadas a cabo por los autores de primera línea en el tema. El conjunto de tales trabajos hace patente la falta de articulación en que se encuentra el constructo «mapa cognitivo». Sin embargo, of recen unas orientaciones a tener en cuenta necesariamente en cualquier futura investigación.

\section{Summary}

Present paper offers a review of researches carried out in the last two decades on cognitive maps on urban areas by most significant authors in that matter. The whole of the researches considered shows the lack of articulation that exists in the construct ucognitive map". Nevertheless, they give some important orientations to be kept in mind for any new research to be done in the future. 


\section{Estudios}

\section{Resumé}

Cet article offre une revision des recherches réalisées pendant les deux dernières décades sur les mappes congitives des zones urbaines par les plus importants auteurs qui ont travaillé sur le theme. L'ensemble des recherches considerés révéle une manque d'articulation dans le construct umappe cognitive». Neanmoins, elles donnent des très profitables orientations a tous ceux qui emtreprennent la réalisation de nouvelles reserches sur le theme. 